\title{
PRÁTICA CLÍNICA DO ENFERMEIRO EM ASSISTÊNCIA CIRCULATÓRIA MECÂNICA: BUSCA DE EVIDÊNCIAS
}

\author{
Nursing clinical practice in mechanical circulatory support: search for evidence \\ La práctica clínica de enfermería en soporte circulatorio mecánico: búsqueda de pruebas
}

Daniela de Araújo Gian Grossi', Marcel Ricardo Chiarini de Oliveira',
Ana Lucia Gargione Galvão de Sant'Anna ${ }^{3}$, Regimar Carla Machado ${ }^{4}$

RESUMO: Objetivo: Analisar na literatura científica a prática clínica do enfermeiro ao paciente em uso de assistência circulatória mecânica. Método: Revisão integrativa da literatura realizada nas bases de dados SciELO, Cochrane Library, PubMed e LILACS, de abril a agosto de 2014. Resultados: Embora haja avanço em pesquisas sobre o tema, ainda há poucos estudos, haja vista oito artigos selecionados. Três artigos apontam para a necessidade do enfermeiro deter o conhecimento sobre assistência circulatória mecânica, para uma assistência eficiente e com qualidade. Dois artigos trouxeram a assistência de enfermagem a pacientes em uso de dispositivos. Três relataram complicações devido ao uso do balão intra-aórtico e mostraram que as complicações podem ser minimizadas a partir da capacitação e da avaliação clínica periódica do enfermeiro. Conclusão: Há poucos estudos direcionados à assistência de enfermagem, havendo a necessidade de maior exploração do tema para subsidiar a prática baseada em evidências cientificas.

Palavras-chave: Enfermagem. Insuficiência cardíaca. Coração auxiliar. Circulação assistida.

ABSTRACT: Objective: To analyze the scientific literature regarding the clinical practice of nurses in patients using mechanical circulatory support. Method: Integrative literature review was conducted from the databases of SciELO, Cochrane Library, PubMed, and LILACS, from April to August 2014. Results: Although there is a progress in the research on this topic, there are only few studies, of which eight articles were selected. Three articles point out the need of the nurses to detain knowledge about mechanical circulatory support for an effective and good service. Two articles approached the nursing care in patients using device. Three studies reported complications because of the use of intra-aortic balloon pump and showed that complications can be minimized through training and periodic clinical assessment of the nurse. Conclusion: There are only few articles that focused on nursing care; therefore, there is a need to further explore this theme to subsidize the practice based on scientific evidence.

Keywords: Nursing. Heart failure. Heart-assist devices. Assisted circulation.

RESUMEN: Objetivo: Analizar en la literatura científica la práctica clínica de las enfermeras a los pacientes utilizándose del soporte circulatorio mecánico. Método: Revisión integradora de la literatura conducida en las bases de datos SciELO, Cochrane Library, PubMed y LILACS, desde abril hasta agosto de 2014. Resultados: Aunque hay avances en la investigación sobre el tema, hay pocos estudios, con solo ocho artículos seleccionados. Tres artículos apuntan a la necesidad de las enfermeras detener los conocimientos sobre soporte circulatorio mecánico para el servicio eficiente y de calidad. Dos artículos presentaron la atención de la enfermería a los pacientes que utilizan dispositivos. Tres estudios informaron complicaciones por la utilización de balón intra-aórtico y mostraron que las complicaciones pueden reducirse al mínimo basándose en la formación y la evaluación clínica periódica de la enfermera. Conclusión: Hay pocos estudios con foco en la atención de la enfermería, y una necesidad de una mayor exploración del tema, para subsidiar la práctica basada en evidencias científicas. Palabras clave: Enfermería. Insuficiencia cardíaca. Corazón auxiliar. Circulación asistida.

'Enfermeira. Especialista em Enfermagem em Cuidados Intensivos com Ênfase em Cardiologia Clínica e Cirúrgica pela Universidade Cruzeiro do Sul (UNICSUL). E-mail: danielagiangrossi@gmail.com Instituito Dante Pazzanese de Cardiologia - Avenida Doutor Dante Pazzanese, 500 - Vila Mariana - CEP: 04012-909 - São Paulo (SP), Brasil.

${ }^{2}$ Enfermeiro. Especialista em Enfermagem em Cuidados Críticos/Cardiologia pela Universidade do Vale do Paraíba (UNIVAP). E-mail: marcel_chiarini@hotmail.com

${ }^{3}$ Enfermeira. Mestre em Engenharia Biomédica pela UNIVAP. Docente do Curso de Especialização em Enfermagem em Cuidados Intensivos com Ênfase em Cardiologia Clínica e Cirúrgica da UNICSUL. E-mail: analuciaggsantanna@gmail.com

${ }^{4}$ Enfermeira. Doutora em Ciências pelo Programa de Pós-graduação em Cirurgia Cardiovascular da Universidade Federal de São Paulo (UNIFESP). Docente do Departamento de Enfermagem da Universidade Federal de São Carlos (UFSCAR). E-mail: regimarmachado@gmail.com

Recebido: 17 nov. 2015 - Aprovado: 23 mar. 2016

DOI: 10.5327/Z1414-4425201600010009 


\section{INTRODUÇÃO}

Reconhecida pela alta taxa de morbidade e mortalidade, a insuficiência cardíaca (IC) é uma síndrome clínica na qual há uma alteração funcional ou estrutural do coração levando à disfunção na ejeção ou acomodação do sangue dentro de valores pressóricos considerados fisiológicos ${ }^{1}$.

Trata-se de uma síndrome com incidência e prevalência elevada no mundo, resultando em 2 milhões de casos a cada ano, com redução da qualidade de vida ${ }^{2}$. Estima-se que 1 a $2 \%$ da população apresente IC nos países desenvolvidos; e em cerca de 10 milhões de pessoas esse diagnóstico está associado à disfunção ventricular na Europa ${ }^{1,2}$.

No Brasil, cerca de 6,4 milhões sofrem de IC, sendo a primeira causa de internação hospitalar em pacientes acima de 60 anos e a terceira causa de internações no Sistema Único de Saúde (SUS) no ano de $2007^{3}$. Os custos com internação por IC chegam a $60 \%$ do custo total do tratamento para essa síndrome ${ }^{3}$.

Ao longo dos anos ocorre um remodelamento ventricular (alterações estruturais, bioquímicas, moleculares e celulares) ${ }^{4-6}$, que pode ser reversível com o uso de dispositivos de assistência circulatória mecânica (ACM), propiciando um remodelamento reverso ${ }^{7}$.

O uso desses dispositivos teve sua eficácia comprovada, quando comparado ao tratamento clínico, no estudo prospectivo e aleatório chamado Rematch ${ }^{8}$. Tal estudo foi realizado com 129 pacientes submetidos ao dispositivo de assistência ventricular esquerda, que apresentavam IC sem indicação de transplante cardíaco ou que o recusaram. Como resultado, observou-se melhor evolução nos pacientes com o dispositivo, ou seja, terapia de destino, em relação aos que utilizaram tratamento exclusivamente clínico.

Quando essa assistência circulatória é utilizada como suporte temporário, em um miocárdio considerado viável, é denominada "ponte ou terapia de resgate". Já nos casos em que o miocárdio é irrecuperável e o transplante cardíaco é crucial, denomina-se "ponte para transplante". Nos casos em que o transplante cardíaco é contraindicado ou quando há recusa do paciente em realizá-lo, denomina-se "terapia de destino"”.

Esse tipo de tratamento tem como objetivos restabelecer a hemodinâmica, melhorar os sintomas por meio do aumento da perfusão de órgãos e prevenir lesão miocárdica, bem como a capacidade funcional dos pacientes ${ }^{10,11}$.

Assim, os dispositivos para o tratamento da IC associada à disfunção ventricular vêm sendo cada vez mais utilizados como ponte para o transplante cardíaco em pacientes com falência miocárdica, bem como na readaptação miocárdica, na restauração do débito cardíaco, na miocardiopatia aguda, no infarto agudo do miocárdio, no choque cardiogênico pós-cardiotomia, na miocardite infecciosa e na rejeição pós-transplante ${ }^{9}$. Salienta-se que o paciente submetido à cirurgia cardíaca (revascularização do miocárdio, troca ou plastia valvar, cirurgia corretora, transplante cardíaco), na maioria das vezes necessita do desvio cardiopulmonar utilizando a máquina de circulação extracorpórea (CEC), no entanto, uma das complicações é a dificuldade em desmamar da CEC, o paciente apresentar baixo débito cardíaco, insuficiência cardíaca intraoperatória e, assim, necessitar inserir uma ACM dentro da sala de operação proporcionando a melhora do débito cardíaco?.

Portanto, os enfermeiros perioperatórios devem buscar o aprimoramento técnico-cientifico para nortear suas ações relacionadas ao uso desses dispositivos, para oferecer maior segurança ao paciente crítico e melhorar a qualidade da assistência prestada, pois os dispositivos podem ser implantados e retirados na sala de operação.

Os dispositivos de ACM podem ser classificados de várias formas, conforme demonstra o Quadro $1^{8,9,11}$.

Por se tratar de um problema de saúde pública e devido aos seus aspectos clínicos e epidemiológicos, bem como à tendência na busca por alternativas para o tratamento da IC e para a preservação da vida, fez-se necessária a realização do presente estudo para subsidiar uma assistência de enfermagem segura, na sala operatória, na recuperação pós-anestésica, na unidade de terapia intensiva e, consequentemente, reduzir a probabilidade de ocorrência de eventos adversos.

\section{OBJETIVO}

Analisar, na literatura científica, a prática clínica do enfermeiro ao paciente em uso de ACM.

Quadro 1. Classificação dos dispositivos de assistência circulatória mecânica.

\begin{tabular}{|c|c|}
\hline Classificação devido a(o) & Dispositivo \\
\hline $\begin{array}{l}\text { Tipo de fluxo: } \\
\text { Contrapulsação } \\
\text { Contínuo } \\
\text { Pulsátil }\end{array}$ & $\begin{array}{l}\text { Balão intra-aórtico } \\
\text { Rolete, centrífuga e axial } \\
\text { Pneumático e elétrico }\end{array}$ \\
\hline Posição em relação ao coração & Série ou paralelo \\
\hline Ventrículo assistido & Direito, esquerdo ou biventricular \\
\hline Grau de substituição ventricular & Total ou parcial \\
\hline Posição em relação ao paciente & Paracorpóreo ou implantável \\
\hline Tempo de permanência & $\begin{array}{c}<30 \text { dias - curta duração } \\
30 \text { dias a } 1 \text { ano - média duração } \\
>1 \text { ano - longa duração }\end{array}$ \\
\hline
\end{tabular}




\section{MÉTODO}

Trata-se de uma revisão integrativa sendo percorridas as seguintes etapas: elaboração da pergunta norteadora e objetivo para a revisão integrativa; estabelecimento de critérios para inclusão e exclusão de estudos (amostragem); definição das informações a serem extraídas dos estudos selecionados (categorização dos estudos); avaliação dos estudos incluídos na revisão integrativa; interpretação dos resultados; apresentação dos resultados (síntese do conhecimento) ${ }^{12}$.

A questão norteadora utilizada para guiar esta revisão integrativa foi: qual a produção científica sobre a prática clínica do enfermeiro a pacientes em uso de ACM?

Na revisão de literatura foram consultadas, no período de abril a agosto de 2014, as bases de dados Scientific Electronic Library Online (SciELO); Cochrane Library; Literatura LatinoAmericana e do Caribe em Ciências da Saúde (LILACS), por meio da Biblioteca Virtual em Saúde (BVS) e PubMed.

Os descritores utilizados, segundo o Medical Subject Headings (MeSH), foram: nursing; heart failure; heart-assist device; ventricle assist device; e segundo os Descritores em Ciências da Saúde (DeCS) foram: insuficiência cardíaca; coração auxiliar; circulação assistida. Para a base de dados PubMed e Cochrane Library, foram utilizados os descritores segundo MeSH. Para a base de dados SciELO, foram utilizados os descritores segundo MeSH e DeCS. Em ambos os casos foi utilizado o operador booleano "AND", executando todas as combinações possíveis entre os descritores.

Os critérios de inclusão foram: artigos originais em português, inglês e espanhol, publicados na íntegra no período de 2005 a 2014; estudos em humanos, adultos (a partir de 19 anos) e idosos; ambos os sexos; revisões sistemáticas, estudos randomizados controlados, estudos com delineamento quase-experimental; estudos que retratassem intervenções, diretrizes ou procedimentos de intervenções de enfermagem em assistência circulatória mecânica.

Os critérios de exclusão incluíram: artigos não disponibilizados na íntegra, dissertações e teses.

Na primeira etapa foram realizadas buscas nas bases de dados e selecionados artigos que atendiam aos objetivos do presente estudo, a partir da leitura dos títulos e resumos.

Foram encontrados 1.413 estudos, sendo 900 da PubMed, 391 da Cochrane, 114 da LILACS e 8 da SciELO. Desses, 742 não estavam disponíveis eletronicamente e 62 eram repetidos. Ainda, poucos atenderam aos critérios de inclusão, sendo selecionados apenas 13, sendo 5 da LILACS, 7 da PubMed e 1 na SciELO. Desses, cinco eram repetidos, restando oito artigos para o presente estudo.
Após essa seleção e a leitura na íntegra dos artigos, utilizouse um instrumento ${ }^{13}$ de coleta adaptado para compilação dos dados coletados, que contemplava os seguintes itens: dados de identificação do artigo original (título, país, idioma, ano de publicação, instituição sede do estudo, periódico), características metodológicas do estudo (tipo, objetivo), avaliação do rigor metodológico, das intervenções mensuradas e dos resultados encontrados.

Os estudos foram analisados por dois revisores independentes e em casos de discordância entre os dois revisores com relação à inclusão de um trabalho, um terceiro revisor enfermeiro foi solicitado. Os revisores eram não cegos.

Utilizou-se o nível de evidência cientifica por tipo de estudo do Oxford Center for Evidence-based Medicine (CEBM) para classificação dos artigos ${ }^{14}$.

\section{RESULTADOS}

Foram selecionados oito artigos, sendo dois publicados em 2013, dois em 2012, um em 2011, dois em 2009 e um em 2006. Em relação ao local de publicação, dois são oriundos do Brasil, seis são oriundos dos Estados Unidos. Quanto aos periódicos, um foi publicado no AORN Journal, outro na AACN Advanced Critical Care, um no Heart Lung, três no Progress in Transplantation, um na Revista Latino Americana de Enfermagem e outro na Acta Paulista de Enfermagem.

Todos os estudos apresentaram nível de evidência 5 , sendo um escore para baixa evidencia cientifica.

A fim de se obter uma análise mais clara dos artigos ${ }^{10,15-19}$ que atenderam aos critérios de inclusão, foram demonstrados na Tabela 1 os artigos selecionados, contendo título, nome dos autores, intervenções estudadas, resultados e conclusões.

\section{DISCUSSÃO}

O presente estudo demonstra que, embora haja avanço em pesquisas sobre ACM, ainda há poucos estudos direcionados à enfermagem, haja vista o número de artigos selecionados para esta revisão integrativa.

Em contrapartida, estudos ${ }^{10,15-19}$ apontam a participação cada vez mais forte dos enfermeiros nos cuidados com ACM. Estudo ${ }^{16}$ enfatiza a presença emergente dos profissionais enfermeiros em programas de ACM nos Estados Unidos, indo de encontro a outro estudo ${ }^{18}$ que aponta as responsabilidades, funções e procedimentos realizados por esses profissionais. 
Tabela 1. Síntese dos artigos selecionados para esta revisão integrativa.

\begin{tabular}{|c|c|c|c|c|}
\hline Título do artigo & Autores & Intervenções estudadas & Resultados & Conclusões \\
\hline $\begin{array}{l}\text { Gastrointestinal } \\
\text { bleeding in } \\
\text { patients with } \\
\text { assist devices: } \\
\text { what every } \\
\text { cardiac nurse } \\
\text { should know }{ }^{10}\end{array}$ & $\begin{array}{l}\text { Ballew CC, } \\
\text { Surrat JF, } \\
\text { Collins TL, } \\
\text { Shah N }\end{array}$ & $\begin{array}{l}\text { Possíveis causas, } \\
\text { o conjunto de } \\
\text { procedimentos } \\
\text { diagnósticos e } \\
\text { tratamentos para } \\
\text { sangramento } \\
\text { gastrintestinal }\end{array}$ & $\begin{array}{l}\text { Incidência de } 13 \text { a } 44 \% \text { dos pacientes } \\
\text { em uso de dispositivos de assistência } \\
\text { ventricular. } 0 \text { sangramento está mais } \\
\text { presente em uso de dispositivo de fluxo } \\
\text { contínuo do que pulsátil. Fator de risco: a } \\
\text { idade avançada. Tratamento: cauterização, } \\
\text { injeção de epinefrina, clipes de metal, } \\
\text { bandagens ou utilização de plasma }\end{array}$ & $\begin{array}{l}\text { Os dispositivos de assistência } \\
\text { ventricular são tratamentos } \\
\text { bem sucedidos a longo prazo, } \\
\text { porém há limitações para } \\
\text { os prestadores de cuidados } \\
\text { pacientes e familiares, uma } \\
\text { vez que esses pacientes } \\
\text { muitas vezes possuem fatores } \\
\text { de risco para sangramento }\end{array}$ \\
\hline $\begin{array}{l}\text { Monitoring } \\
\text { patients with } \\
\text { continuous-flow } \\
\text { ventricular assist } \\
\text { devices outside } \\
\text { of the intensive } \\
\text { care unit: novel } \\
\text { challenges to } \\
\text { beside nursing }{ }^{15}\end{array}$ & $\begin{array}{l}\text { O'Shea G, } \\
\text { Teuteberg } \\
\text { JJ, } \\
\text { Severy } \\
\text { DA }\end{array}$ & $\begin{array}{l}\text { Descreve a função } \\
\text { de dispositivos de } \\
\text { fluxo contínuo e } \\
\text { como isso afeta a } \\
\text { função de opções } \\
\text { de monitoramento, } \\
\text { e a forma de avaliar } \\
\text { clinicamente para } \\
\text { identificar rapidamente } \\
\text { aqueles cuja condição } \\
\text { pode se deteriorar }\end{array}$ & $\begin{array}{l}\text { Apesar de todos os avanços tecnológicos, o } \\
\text { dispositivo de monitoração mais importante } \\
\text { ainda é o "enfermeiro de cabeceira" }\end{array}$ & $\begin{array}{l}\text { A condição de cada paciente } \\
\text { deve ser avaliada de acordo } \\
\text { com sua clínica, sendo o } \\
\text { acompanhamento de dados } \\
\text { uma informação auxiliar } \\
\text { para observar }\end{array}$ \\
\hline $\begin{array}{l}\text { Practice pattern } \\
\text { and professional } \\
\text { nurse } \\
\text { practitioners } \\
\text { in mechanical } \\
\text { circulatory } \\
\text { support } \\
\text { programs in the } \\
\text { United States: } a \\
\text { survey report }\end{array}$ & $\begin{array}{l}\text { Casida JM, } \\
\text { Pastor J }\end{array}$ & $\begin{array}{l}\text { Padrão de prática e } \\
\text { questões profissionais } \\
\text { enfrentados pela } \\
\text { enfermagem na } \\
\text { evolução de programas } \\
\text { de suporte circulatório } \\
\text { mecânico nos Estados } \\
\text { Unidos }\end{array}$ & $\begin{array}{l}\text { A maioria dos enfermeiros possuía } \\
\text { especialidade de cirurgia cardíaca ou } \\
\text { cuidados intensivos e não de transplante; } \\
90 \% \text { foram autorizados a prescrever } \\
\text { medicamentos e procedimentos e } 64 \% \\
\text { autorizados a darem alta hospitalar; } 96 \% \\
\text { caracterizam o trabalho como estressante. } \\
\text { Muitos apontaram falta de conhecimento da } \\
\text { prática baseada em evidência ou diretrizes. } \\
\text { Apenas } 2 \text { dos } 48 \text { entrevistados disseram } \\
\text { estar satisfeitos com o trabalho. }\end{array}$ & $\begin{array}{l}\text { Embora os resultados sejam } \\
\text { de natureza preliminar, } \\
\text { os dados expandiram } \\
\text { a informação existente } \\
\text { desse importante grupo de } \\
\text { profissionais de enfermagem, } \\
\text { proporcionando um roteiro } \\
\text { para futuras pesquisas e } \\
\text { desenvolvimento de políticas } \\
\text { relevantes para programas } \\
\text { ACM nos Estados Unidos }\end{array}$ \\
\hline $\begin{array}{l}\text { Complicações } \\
\text { do balão intra- } \\
\text { aórtico em } \\
\text { uma coorte } \\
\text { de pacientes } \\
\text { hospitalizados: } \\
\text { implicações para } \\
\text { a assistência de } \\
\text { enfermagem }\end{array}$ & $\begin{array}{l}\text { Assis RBS, } \\
\text { Azzolin K, } \\
\text { Boaz M, } \\
\text { Rabelo ER }\end{array}$ & $\begin{array}{l}\text { Complicações } \\
\text { decorrentes da } \\
\text { utilização do BIA, } \\
\text { relacionando-as } \\
\text { com o tempo de } \\
\text { permanência, com a } \\
\text { presença de fatores de } \\
\text { risco/comorbidades e } \\
\text { com os registros de } \\
\text { enfermagem }\end{array}$ & $\begin{array}{l}\text { A média de permanência com o BIA foi de } 28 \mathrm{~h} \text {; } \\
25 \% \text { dos pacientes apresentou complicações } \\
\text { vasculares. } 0 \text { sexo masculino apresentou } \\
\text { mais complicações. Quanto maior o número } \\
\text { de horas com o BIA, maior o número de } \\
\text { complicações. Apenas } 68,3 \% \text { dos prontuários } \\
\text { relataram o uso do cateter; Apenas } 28,8 \% \\
\text { apresentaram evolução descrevendo a } \\
\text { presença do dispositivo e } 26,9 \% \text { relataram as } \\
\text { condições do paciente após sua retirada. }\end{array}$ & $\begin{array}{l}\text { As complicações mais } \\
\text { frequentes foram as } \\
\text { vasculares. Pacientes } \\
\text { com mais de } 37 \mathrm{~h} \text { com o } \\
\text { dispositivo apresentaram } \\
\text { mais complicações. } \\
\text { A ocorrência das complicações } \\
\text { pode ser minimizada pela } \\
\text { avaliação clínica periódica e } \\
\text { monitorização laboratorial }\end{array}$ \\
\hline $\begin{array}{l}\text { A survey of } \\
\text { nurses in } \\
\text { themechanical } \\
\text { circulatory } \\
\text { support } \\
\text { programs in the } \\
\text { United States }\end{array}$ & $\begin{array}{l}\text { Casida JM, } \\
\text { Ilacqua J }\end{array}$ & $\begin{array}{l}\text { O papel dos } \\
\text { enfermeiros em } \\
\text { programas de suporte } \\
\text { circulatório mecânico } \\
\text { nos Estados Unidos }\end{array}$ & $\begin{array}{l}\text { Um total de } 63 \% \text { dos enfermeiros registrados } \\
\text { não possuíam especialidade. Mais de } 62 \% \text { de } \\
\text { seus trabalhos foram destinados à assistência } \\
\text { direta ao paciente e seus cuidadores. Menos } \\
\text { da metade dos participantes necessitaram } \\
\text { completar o suporte básico ou avançado } \\
\text { de vida. Um total de } 71 \% \text { obtiveram seu } \\
\text { conhecimento por meios informais. A função } \\
\text { mais comum no trabalho foi a coordenação } \\
\text { dos cuidados. Tanto o EPA quanto ER exercem } \\
\text { função de educadores em todo serviço de } \\
\text { saúde; } 96 \% \text { dos ER percebe melhora na } \\
\text { qualidade de vida do paciente enquanto } \\
\text { apenas } 90 \% \text { dos EPA referiram. }\end{array}$ & $\begin{array}{l}\text { Independentemente do seu } \\
\text { nível de autonomia na prática } \\
\text { clínica, esses enfermeiros } \\
\text { são consultores, educadores, } \\
\text { pesquisadores e líderes, além } \\
\text { de coordenar a assistência ao } \\
\text { paciente }\end{array}$ \\
\hline
\end{tabular}


Tabela 1. Continuação.

\begin{tabular}{|c|c|c|c|c|}
\hline Título do artigo & Autores & Intervenções estudadas & Resultados & Conclusões \\
\hline $\begin{array}{l}\text { Validação de } \\
\text { protocolo para } \\
\text { assistência } \\
\text { a pacientes } \\
\text { com balão } \\
\text { intra-aórtico }\end{array}$ & $\begin{array}{l}\text { Machado } \\
\text { RC, } \\
\text { Guerra } \\
\text { GM, } \\
\text { Branco } \\
\text { JNR }\end{array}$ & $\begin{array}{l}\text { Elaborar um protocolo } \\
\text { de cuidados a pacientes } \\
\text { com BIA e validar } \\
\text { o conteúdo desse } \\
\text { protocolo }\end{array}$ & $\begin{array}{l}\text { Foram avaliados } 36 \text { itens direcionados aos } \\
\text { cuidados dos pacientes em uso de BIA. Ao } \\
\text { final elaborou-se um protocolo com } 22 \text { itens } \\
\text { que percorrem desde a educação do paciente } \\
\text { e família até a realização do procedimento e } \\
\text { cuidados até sua retirada }\end{array}$ & $\begin{array}{l}\text { Elaborado um protocolo } \\
\text { com } 22 \text { itens referentes aos } \\
\text { cuidados a pacientes com BIA }\end{array}$ \\
\hline $\begin{array}{l}\text { Intra-aortic } \\
\text { baloon pump } \\
\text { therapy: a primer } \\
\text { for perioperative } \\
\text { nurses }^{20}\end{array}$ & $\begin{array}{l}\text { Tremper } \\
\text { RS }\end{array}$ & $\begin{array}{l}\text { Uso de balão } \\
\text { intra-aórtico no } \\
\text { perioperatório }\end{array}$ & $\begin{array}{l}\text { Explicita indicações, contraindicações } \\
\text { e complicações, uso perioperatório, } \\
\text { manipulação e configuração do console } \\
\text { e fisiologia }\end{array}$ & $\begin{array}{l}\text { Ter um conhecimento do BIA } \\
\text { permite aos enfermeiros } \\
\text { antecipar as necessidades da } \\
\text { equipe cirúrgica e melhorar os } \\
\text { resultados dos pacientes }\end{array}$ \\
\hline $\begin{array}{l}\text { Ventricular assist } \\
\text { devices: what } \\
\text { intensive care } \\
\text { unit nurses need } \\
\text { to know about } \\
\text { postoperative } \\
\text { management }{ }^{21}\end{array}$ & O'Shea G & $\begin{array}{l}\text { Estratégias de gestão } \\
\text { de enfermagem no } \\
\text { pós-operatório e } \\
\text { complicações mais } \\
\text { importantes que os } \\
\text { enfermeiros de cuidados } \\
\text { críticos precisam saber }\end{array}$ & $\begin{array}{l}\text { O enfermeiro deve atentar-se a } \\
\text { monitorização, hemorragia, disfunção do } \\
\text { ventrículo direito, tamponamento cardíaco, } \\
\text { obstrução da cânula, disritmias, disfunção } \\
\text { orgânica secundária, hemólise, disfunção } \\
\text { respiratória e neurológica, infecção, falhas } \\
\text { mecânicas, trombose }\end{array}$ & $\begin{array}{l}\text { Aponta a necessidade do } \\
\text { conhecimento por parte } \\
\text { dos enfermeiros e que esse } \\
\text { dispositivo melhora a qualidade } \\
\text { de vida comparado com terapias } \\
\text { medicamentosas, porém, } \\
\text { apresenta riscos e complicações }\end{array}$ \\
\hline
\end{tabular}

BIA: balão intra-aórtico; EPA: enfermeiras de práticas avançadas; ER: enfermeiros registrados; ACM: assistência circulatória mecânica.

Dentre os 8 estudos selecionados, $3(37,5 \%)^{15,17}$ apontam para a necessidade do enfermeiro ter posse do conhecimento a respeito da ACM utilizada, a fim de se obter resultados de qualidade e mais rápidos, tanto aos pacientes quanto à equipe multiprofissional. Ressalta-se que, em uma das pesquisas ${ }^{18}$, há o dado de que $75 \%$ dos enfermeiros que atuam com o programa de ACM dos Estados Unidos tiveram aulas sobre manejo dos dispositivos, porém, grande parte dos enfermeiros obteve seu conhecimento por meios informais (out of job). Estudos trazem que os enfermeiros devem estar capacitados quanto às indicações, benefícios, riscos e possíveis complicações relacionadas a essa terapêutica, ressaltando como estratégia de cuidado o exame físico acurado ${ }^{16,17,19}$.

Dois artigos (25\%) trouxeram a assistência de enfermagem em pacientes em uso de dispositivo de assistência ventricular ${ }^{10,15}$, os quais demonstram os desafios enfrentados pelos enfermeiros principalmente quando utilizado o dispositivo de fluxo contínuo, uma vez que não é possível aferir os parâmetros hemodinâmicos através dos métodos invasivos tradicionais. Dessa forma, o profissional deve ser capacitado a identificar complicações desses dispositivos — como o sangramento gastrointestinal, que ainda não possui uma causa clara - e a instituição deve oferecer recursos a fim de que seja possível o uso de um ultrassom Doppler para verificação da pressão sanguínea, por exemplo.

Três estudos $(37,5 \%)^{10,17,19}$ tratam do uso do balão intra aórtico (BIA) e todos mostraram que as complicações podem ser minimizadas a partir da capacitação e da avaliação clínica periódica do enfermeiro. Um estudo ${ }^{19}$ aponta que de 104 prontuários analisados, apenas $28,8 \%$ trouxeram evolução descrevendo o uso do BIA e $26,9 \%$ relataram, após a retirada do dispositivo, as condições do paciente, enfatizando que essa deficiência nos registros pode estar associada às atribuições técnicas e administrativas, bem como ao número de pacientes a serem assistidos. Essa informação vai de encontro a um dos estudos selecionados para esta revisão integrativa ${ }^{16}, \mathrm{o}$ qual explora as atribuições e responsabilidades em excesso nesse grupo de profissionais, sendo que $96 \%$ dos enfermeiros caracterizaram o trabalho como estressante, apontando ainda a falta de apoio institucional e falta de reconhecimento profissional, pois apenas $2(4,16 \%)$ dos 48 entrevistados relataram estarem satisfeitos com a profissão.

Um desses estudos enfatiza a importância dos enfermeiros de centro cirúrgico possuírem conhecimentos básicos sobre o BIA, por serem os responsáveis pela resolução dos problemas com o dispositivo. Também descreve detalhadamente as atividades desempenhadas pelo enfermeiro em todas as etapas do procedimento (preparo do material/equipamento, implantação do dispositivo e remoção), assim como as indicações, contraindicações e complicações dessa complexa terapia. Tais complicações podem ser decorrentes das infecções, da isquemia do membro, da ruptura do balão, dos sangramentos, da paraplegia ou das dores abdominais ocasionadas pela oclusão da artéria mesentérica ${ }^{19}$.

Grande parte das complicações dos estudos apresentados nesta revisão ${ }^{10,14,15,17}$ poderiam ser minimizadas ou evitadas 
com o uso de protocolos, uma vez que esses norteiam procedimentos clínicos, fluxos e condutas, aumentando a probabilidade de resultados de qualidade ${ }^{17,18}$.

Estudo $^{19}$ desenvolveu um protocolo de cuidados ao paciente em uso de BIA, o qual é indicado para auxiliar ou reabilitar o fluxo coronariano. Tal protocolo objetivou diminuir os fatores de risco da terapêutica e trazer evidências para a prática clinica do enfermeiro. Destaca 22 itens relacionados à assistência ao paciente em uso do BIA, tais como o preenchimento do transdutor do sistema com heparina, a antissepsia da pele na região de inserção do dispositivo com clorexidina a $2 \%$, manter o paciente em decúbito dorsal horizontal, após a inserção do dispositivo, com o membro restringido para evitar flexão e prevenir obstrução do fluxo e hematomas, avaliar periodicamente o membro, comprimir manualmente o local após a retirada do dispositivo e realizar curativo compressivo $^{19}$. Dessa forma, oferece subsídios para o enfermeiro propor intervenções almejando uma assistência de qualidade ao paciente crítico.

A presença, cada vez frequente, de pacientes críticos no centro cirúrgico evidencia a necessidade de enfermeiros tecnologicamente capacitados para o atendimento dessas complexas terapias e o tamanho da responsabilidade desses profissionais.

Dessa forma, a fim de se obter capacitação dos profissionais, qualidade na assistência e, consequentemente, melhores resultados aos pacientes em uso de dispositivos de ACM, se deve voltar o olhar para a sistematização do processo de enfermagem, a qual permite desenvolver metodologias de cuidado interdisciplinares, guiando o processo de trabalho da enfermagem ${ }^{22}$.

\section{CONCLUSÃO}

Há poucos estudos direcionados à assistência de enfermagem, havendo a necessidade de maior exploração do tema para subsidiar a prática baseada em evidências cientificas. Apenas dois estudos trouxeram apontamento sobre a prática clínica do enfermeiro em ACM. Os demais enfatizam sobre a necessidade do enfermeiro deter conhecimento e a importância da atualização para uma assistência de qualidade. Dessa forma, foi possível concluir que grande parte dos enfermeiros que estão na prática da ACM acabam obtendo o conhecimento no dia a dia profissional.

Além disso, o artigo enfatiza a necessidade de instituições trabalharem com as metodologias de cuidado e protocolos, por serem as conquistas mais importantes na assistência de enfermagem com a finalidade de se obter maior capacitação dos profissionais, melhor qualidade da assistência, otimização do tempo de trabalho e planejamento institucional para fornecer recursos aos profissionais.

\section{REFERÊNCIAS}

1. McMurray JJV, Adamopoulos S, Anker SD, Auricchio A, Böhm M, Dickstein K, et al. ESC Guidelines for the diagnosis and treatment of acute and chronic heart failure 2012: the Task Force for the Diagnosis and Treatment of Acute and Chronic Heart Failure 2012 of the European Society of Cardiology. Developed in collaboration with the Heart Failure Association (HFA) of the ESC. Eur Heart J. 2012;33(14):1787-847.

2. Hunt SA, Abraham WT, Chin MH, Feldman AM, Francis GS, Ganiats TG, et al. 2009 Focused update incorporated into the ACC/AHA 2005 Guidelines for the Diagnosis and Management of Heart Failure in Adults: a report of the American College of Cardiology Foundation/ American Heart Association Task Force on Practice Guidelines. Developed in collaboration with the International Society for Heart and Lung Transplantation. Circulation. 2009;119(14):391-479.

3. Brasil. Ministério da Saúde. DATASUS. Informações de Saúde. Morbidade hospitalar do SUS [Internet]. Brasília: Ministério da Saúde;
2012 [acesso em 2012 abr 29]. Disponível em: http://tabnet.datasus. gov.br/cgi/deftohtm.exe?sih/cnv/niuf.def

4. Ertl G, Ruschitzka F. The year in cardiology 2013: heart failure. Eur Heart J. 2014;35(7):470-3.

5. Anderson KM. Discharge clinical characteristics and 60-day readmission in patients hospitalized with heart failure. J Cardiovasc Nurs. 2014;29(3):232-41.

6. Gallagher R. Self management, symptom monitoring and associated factors in people with heart failure living in the community. Eur $\mathrm{J}$ Cardiovasc Nurs. 2010;9(3):153-60.

7. HeXW, Song ZZ. Evaluation of left ventricular function, rotation, twist and untwist in patients with hypertrophic cardiomyopathy. Exp Clin Cardiol. 2013;18(1):e47-9. 
8. Rose EA, Gelijns AC, Moskowitz AJ, Heitjan DF, Stevenson LW, Dembitsky W, et al. Long-term use of a left ventricular assist device for end-stage heart failure. N Engl J Med. 2001;345(20):1435-43.

9. D'Alessandro C, Aubert S, Golmard JL, Praschker BL, Luyt CE, Pavie $A$, et al. Extra-corporeal membrane oxygenation temporary support for early graft failure after cardiac transplantation. Eur J Cardiothorac Surg. 2010;37(2):343-9.

10. Ballew CC, Surrat JF, Collins TL, Shah N. Gastrointestinal bleeding in patients with ventricular assist devices: what every cardiac nurse should know. Prog Transplant. 2013;23(3):229-34.

11. John R, Long JW, Massey HT, Griffith BP, Sun BC, Tector AJ, et al. Outcomes of a multicenter trial of the Levitronix CentriMag ventricular assist system for short-term circulatory support. J Thorac Cardiovasc Surg. 2011;141(4):932-9.

12. Mendes KDS, Silveira RCCP, Galvão CM. Revisão integrativa: método de pesquisa para incorporação de evidências na saúde e na enfermagem. Texto Contexto Enferm. 2008;17(4):758-64.

13. Ursi ES, Galvão CM. Prevenção de lesões de pele no perioperatório: revisão integrativa da literatura. Rev Latino-am Enfermagem. 2006;14(1):124-31.

14. Phillips B, Ball C, Sackett D et al. Oxford Centre for Evidence-based Medicine - Levels of Eviidence (March, 2009) [acesso em 2015 abr 19]. Disponível em: http://www.cebm.net/oxford-centre-evidencebased-medicine-levels-evidence-march-2009/.
15. O'Shea G, Teuteberg JJ, Severyn DA. Monitoring patients with continuousflow ventricular assist devices outside of the intensive care unit: novel challenges to bedside nursing. Prog Transplant. 2013;23(1):39-46.

16. Casida JM, Pastor J. Practice pattern and professional issues of nurse practitioners in mechanical circulatory support programs in the United States: a survey report. Prog Transplant. 2012;22(3):229-36.

17. Assis RBS, Azzolin K, Boaz M, Rabelo ER. Complicações do balão intra-aórtico em uma coorte de pacientes hospitalizados: implicações para a assistência de enfermagem. Rev Latino-am Enfermagem. 2009; 17(5):658-63.

18. Casida JM, Ilacqua J. A survey of nurses in the mechanical circulatory support programs in the United States. Heart Lung. 2011;40(4):e103-11.

19. Machado RC, Guerra GM, Branco JNR. Validação de protocolo para assistência a pacientes com balão intra-aórtico. Acta Paul Enferm. 2012;25(No. Esp 1):13-9.

20. Tremper RS. Intra-aortic baloon pump therapy: a primer for perioperative nurses. AORN J. 2006;84(1):34-44.

21. O'Shea G. Ventricular assist devices: what intensive care unit nurses need to know about postoperative management. AACN Adv Crit Care. 2012;23(1):69-83.

22. Lopes MVO, Silva VM, Araujo TL. Validação de diagnósticos de enfermagem: desafios e alternativas. Rev Bras Enferm. 2013;66(5):649-55 